Mean-field equations for cigar- and disc-shaped Bose and Fermi superfluids

This content has been downloaded from IOPscience. Please scroll down to see the full text. 2009 J. Phys. B: At. Mol. Opt. Phys. 42215306

(http://iopscience.iop.org/0953-4075/42/21/215306)

View the table of contents for this issue, or go to the journal homepage for more

Download details:

IP Address: 134.58.253.57

This content was downloaded on 09/04/2014 at 12:57

Please note that terms and conditions apply. 


\title{
Mean-field equations for cigar- and disc-shaped Bose and Fermi superfluids
}

\author{
Camilo A G Buitrago and S K Adhikari \\ Instituto de Física Teórica, UNESP_São Paulo State University, 01.140-070 São Paulo, Brazil \\ E-mail: adhikari@ift.unesp.br
}

Received 5 June 2009, in final form 4 September 2009

Published 14 October 2009

Online at stacks.iop.org/JPhysB/42/215306

\begin{abstract}
Starting from the three-dimensional (3D) time-dependent nonlinear Gross-Pitaevskii equation for a Bose-Einstein condensate (BEC) and the density-functional (DF) equation for a Fermi superfluid at the unitarity and Bardeen-Cooper-Schrieffer (BCS) limits, we derive effective one- (1D) and two-dimensional (2D) mean-field equations, respectively, for the dynamics of a trapped cigar- and disc-shaped BEC and Fermi superfluid by using the adiabatic approximation. The reduced 1D and 2D equations for a cigar- and disc-shaped Fermi superfluid have simple analytic non-linear terms and at unitarity produce results for stationary properties and non-stationary breathing oscillation and free expansion in excellent agreement with the solution of the full 3D equation.
\end{abstract}

(Some figures in this article are in colour only in the electronic version)

\section{Introduction}

Many of the stationary and non-stationary properties of a trapped Bose-Einstein condensate (BEC) (or a Bose superfluid) can be satisfactorily explained by the mean-field Gross-Pitaevskii (GP) equation [1]. However, there is no such mean-field equation in configuration space for the Fermi superfluid even after the experimental realization [2-5] of the crossover from the Bardeen-Cooper-Schrieffer (BCS) limit through unitarity to the Bose limit in a trapped Fermi superfluid.

To study the properties of stationary and non-stationary states of a trapped Fermi superfluid with an equal number of paired spin-up and -down fermions, Adhikari and Salashnich developed a Galilei-invariant non-linear density-functional (DF) equation valid from the weak-coupling BCS limit to unitarity $[6,7]$. The solution of the DF equation of [6] yielded results for the energy of a trapped Fermi superfluid which are in close agreement with those obtained from Monte Carlo calculations [8] not only in the BCS and unitarity limits but also along the BCS-unitarity crossover [2].

In actual experiments, an axially symmetric $[1,3,9]$, rather than a spherical, trap is usually employed for the confinement of the BEC or Fermi superfluid. In many situations of actual interest, the trap has an extreme geometry [9], e.g. very strong radial or axial confinement. Consequently, the superfluid is formed in the shape of a cigar or a disc and the solution of the non-linear equation in such cases deserves special attention. The cigar-shaped BEC or Fermi superfluid is quasi-one-dimensional (1D) and the reduction of the full threedimensional (3D) equation to accurate 1D form would be of advantage. Similarly, it would be beneficial to reduce the full 3D equation to $2 \mathrm{D}$ form for the description of a disc-shaped BEC and Fermi superfluid.

We propose a simple scheme for the 3D-1D and 3D$2 \mathrm{D}$ reduction of the GP equation for a BEC and the DF equation for a Fermi superfluid at the BCS and unitarity limits respectively. The 3D-1D reduction for a cigar-shaped BEC or a Fermi superfluid is done in the adiabatic approximation which assumes that the essential dynamics to be confined in the axial direction with the radial degrees of freedom adjusting instantaneously to the minimum energy equilibrium configuration compatible with the axial dynamics $[10,11]$. Under this approximation the $3 \mathrm{D}$ wave-function factorizes into an explicitly time-dependent axial and time-independent radial parts which allows for a formal reduction of the original timedependent 3D non-linear equation into a time-dependent 1D and an auxiliary time-independent $2 \mathrm{D}$ equations. The same is also true for a disc-shaped BEC or a Fermi superfluid with the role of time-dependent $1 \mathrm{D}$ and auxiliary time-independent 2D equations interchanged, e.g. the time-dependent equation is now $2 \mathrm{D}$ and the auxiliary equation $1 \mathrm{D}$ in nature. 
First we illustrate the present scheme through an application to a BEC described by the GP equation, where there are already several schemes [10-19] for 3D-1D and 3D-2D reductions. Among these, the reduction schemes of Salasnich et al (SPR) [10, 12] and Muñoz Mateo et al (MMD) $[11,13]$ are the simplest to implement and have been shown to be the most efficient [11]. We compare the present scheme with those of SPR [10, 12] and MMD [11, 13] for a BEC. Recently, Adhikari and Salasnich (AS) [20] suggested one scheme for such 3D-1D and 3D-2D reductions of the 3D DF equation for a Fermi superfluid at the BCS and unitarity limits. We apply our scheme to a Fermi superfluid and obtain distinct $3 \mathrm{D}-1 \mathrm{D}$ and $3 \mathrm{D}-2 \mathrm{D}$ reductions.

In section 2 we illustrate the 3D-1D and 3D-2D reduction schemes in the case of the GP equation for a BEC. In section 3 we consider the reduction schemes for the non-linear DF equation of a Fermi superfluid at unitarity and BCS limits. In this case we reduce the 3D DF equation to $1 \mathrm{D}$ (for cigarshaped superfluid) and 2D (for disc-shaped superfluid) forms with analytic non-linear terms. We show that the reduced 1D and 2D equations for cigar- and disc-shaped Fermi superfluids are very effective for describing the stationary states and nonstationary breathing oscillation and free expansion of the Fermi superfluid at unitarity in close agreement with the solution of the full 3D DF equation. Finally, in section 4 we give a summary and some concluding remarks.

\section{3D-1D and 3D-2D reductions of the GP equation}

We first apply our approach to a BEC described by the GP equation. The GP equation for $N$ bosons of mass $m$ is written as [1]

$$
\left[-\mathrm{i} \hbar \frac{\partial}{\partial \hat{t}}-\frac{\hbar^{2} \nabla_{\hat{\mathbf{r}}}^{2}}{2 m}+V(\hat{\mathbf{r}})+\frac{4 \pi \hbar^{2} \hat{a} N}{m}|\Psi(\mathbf{r})|^{2}\right] \Psi(\hat{\mathbf{r}}, t)=0,
$$

with $\hat{a}$ the atomic scattering length, normalization $\int|\Psi|^{2} \mathrm{~d}^{3} \hat{\mathbf{r}}=1$, and $V(\hat{\mathbf{r}})=m \omega^{2}\left(\hat{\rho}^{2}+\lambda^{2} \hat{z}^{2}\right) / 2$ the harmonic trapping potential with frequencies $\omega$ and $\lambda \omega$ in $\operatorname{radial}(\hat{\rho})$ and axial $(\hat{z})$ directions $(\hat{\mathbf{r}} \equiv \hat{\rho}, \hat{z})$ respectively. Employing dimensionless harmonic oscillator units $t=$ $\hat{t} \omega, \mathbf{r}=\hat{\mathbf{r}} / a_{\rho}, z=\hat{z} / a_{\rho}, \rho=\hat{\rho} / a_{\rho}, a=\hat{a} / a_{\rho}, \psi=$ $\Psi a_{\rho}^{3 / 2}, a_{\rho}=\sqrt{\hbar / m \omega},(1)$ can be written as

$\left[-\mathrm{i} \frac{\partial}{\partial t}-\frac{\nabla_{\mathbf{r}}^{2}}{2}+\frac{\rho^{2}+\lambda^{2} z^{2}}{2}+4 \pi a N|\psi|^{2}\right] \psi(\mathbf{r}, t)=0$,

with the normalization $\int|\psi|^{2} \mathrm{~d}^{3} \mathbf{r}=1$.

In the limits of very small (cigar-shaped trap) and very large (disc-shaped trap) $\lambda$, the length scales in the axial and radial directions are very different. Consequently, the correlations between these two directions can be neglected and the condensate wave-function could be factorized in variables $\rho$ and $z[10,11]$. In the case of cigar-shaped traps the dynamics takes place in the axial direction. The opposite happens in the case of a disc-shaped trap. For a stationary solution $\psi(\mathbf{r})$ of (2) in a cigar-shaped trap one can define a linear density $\phi^{2}(z) \equiv \int \mathrm{d}^{2} \rho \psi^{2}(\mathbf{r})$. Similarly, for a disc-shaped trap one can define a radial density $\varphi^{2}(\rho) \equiv \int \mathrm{d} z \psi^{2}(\mathbf{r})$.

\subsection{D-1D reduction for a cigar-shaped BEC}

For a cigar-shaped trap, the above consideration leads to the factorization [11]

$$
\psi(\mathbf{r}, t)=\varphi\left(\rho, n_{1}(z, t)\right) \phi(z, t),
$$

where linear density $n_{1}$ is defined as $n_{1}(z, t) \equiv N|\phi(z, t)|^{2}=$ $N \int \mathrm{d}^{2} \rho|\psi|^{2}$ and normalizations $\int \mathrm{d}^{2} \rho\left|\varphi\left(\rho, n_{1}\right)\right|^{2}=$ $\int \mathrm{d} z|\phi(z, t)|^{2}=1$. We assume that the function $\varphi\left(\rho, n_{1}(z, t)\right)$ has no explicit $t$ or $z$ dependence and hence these derivatives do not act on this function. This is a good approximation, in general, as we find in numerical studies. The substitution of (3) into (2) then leads to

$$
\begin{aligned}
& \varphi\left(\rho, n_{1}\right)\left[\mathrm{i} \frac{\partial}{\partial t}+\frac{1}{2} \frac{\partial^{2}}{\partial z^{2}}-\frac{1}{2} \lambda^{2} z^{2}\right] \phi(z, t) \\
& =\phi(z, t)\left[-\frac{1}{2} \nabla_{\rho}^{2}+\frac{1}{2} \rho^{2}+4 \pi a n_{1}|\varphi|^{2}\right] \varphi\left(\rho, n_{1}\right) .
\end{aligned}
$$

Multiplying (4) by $\varphi^{*}\left(\rho, n_{1}\right)$ and integrating in $\rho$, this equation can be rewritten as [11]

$$
\begin{aligned}
& {\left[\mathrm{i} \frac{\partial}{\partial t}+\frac{1}{2} \frac{\partial^{2}}{\partial z^{2}}-\frac{1}{2} \lambda^{2} z^{2}-\mu_{\rho}\left(n_{1}\right)\right] \phi(z, t)=0,} \\
& \mu_{\rho}\left(n_{1}\right)=\int \mathrm{d}^{2} \rho \varphi^{*}\left[-\frac{1}{2} \nabla_{\rho}^{2}+\frac{1}{2} \rho^{2}+4 \pi a n_{1}|\varphi|^{2}\right] \varphi,
\end{aligned}
$$

where $\mu_{\rho}\left(n_{1}\right)$ is the chemical potential emerging from the $2 \mathrm{D}$ GP equation:

$\left[-\frac{1}{2} \nabla_{\rho}^{2}+\frac{1}{2} \rho^{2}+4 \pi a n_{1}|\varphi|^{2}-\mu_{\rho}\left(n_{1}\right)\right] \varphi\left(\rho, n_{1}\right)=0$.

We have decoupled the essential axial $(z)$ and non-essential radial $(\rho)$ degrees of freedom. The solution of the timeindependent radial GP equation (7) leads to the chemical potential $\mu_{\rho}\left(n_{1}\right)$ given by (6), which is the non-linear term of the axial GP equation (5).

The form of the chemical potential $\mu_{\rho}\left(n_{1}\right)$ of (7) is known in the small and large $N$ limits. In the small $N$ weak-coupling limit, the wave-function can be approximated by the following normalized Gaussian form [21]:

$$
\varphi\left(\rho, n_{1}\right)=\exp \left[-\rho^{2} /\left(2 \alpha^{2}\right)\right] /\left(\sqrt{\alpha^{2} \pi}\right),
$$

where $\alpha$ is the width. With this wave form the chemical potential of (6) becomes

$$
\mu_{\rho}\left(n_{1}\right)=\left(\frac{\alpha^{2}}{2}+\frac{1}{2 \alpha^{2}}\right)+\frac{2 a n_{1}}{\alpha^{2}} .
$$

In the large $N$ Thomas-Fermi (TF) limit, as $a n_{1} \rightarrow \infty$, the kinetic energy gradient operator in (7) can be neglected and this equation has an analytic solution. The normalization condition of the TF wave-function leads to $[10,11]$

$$
\mu_{\rho}\left(n_{1}\right)=\sqrt{4 a n_{1}} \text {. }
$$

We suggest the following simple interpolation formula for $\mu_{\rho}\left(n_{1}\right)$ valid for small to large $a n_{1}$ incorporating the limiting values (9) and (10)

$$
\mu_{\rho}\left(n_{1}\right)=\frac{1}{2 \alpha^{2}}-\frac{\alpha^{2}}{2}+\sqrt{\alpha^{4}+4 a n_{1}}
$$


Table 1. Chemical potential $\mu_{\rho}\left(n_{1}\right)$ of (6) for different $a n_{1}$ obtained from an accurate numerical solution of (7), and from (11) (present, $\alpha=0.985$ ), (12) (MMD) [11] and (13) (SPR) [10].

\begin{tabular}{ccclc}
\hline$a n_{1}$ & Numerical & MMD & SPR & Present \\
\hline 0 & 1 & 1 & 1 & 1.00045 \\
0.2 & 1.346427 & 1.34164 & 1.35225 & 1.34983 \\
1 & 2.257135 & 2.23607 & 2.30940 & 2.25314 \\
10 & 6.432456 & 6.40312 & 6.76475 & 6.42877 \\
100 & 20.04320 & 20.0250 & 21.2309 & 20.0538 \\
\hline
\end{tabular}

to be used in (5), where $\alpha$ is taken as a fixed constant for all $n_{1}$. In the weak-coupling $a n_{1} \rightarrow 0$ limit, (7) reduces to the Schrödinger equation for a 2D harmonic oscillator with the exact solution (8) with $\alpha=1$. Then (11) is a good approximation to (6) for $\alpha=1$. For slightly larger values of $a n_{1}$ (11) continues to be a good approximation to (6), but with a slightly smaller value of $\alpha$. Motivated by this, we take a slightly smaller value of $\alpha$ in (11). For large $a n_{1},(11)$ has the correct TF limit independent of the value of $\alpha$ employed. This flexibility in introducing a width $\alpha \approx 1$ (slightly different from $\alpha=1$ ) in (11) will be fundamental in making the 1D model equation (5) a faithful approximation to the 3D GP equation (2) for a cigar-shaped condensate for all $a n_{1}$.

By construction, approximation (11) satisfies the weakcoupling and TF limits (9) and (10), respectively, for small and large $a n_{1}$. The approximation of MMD is [11]

$$
\mu_{\rho}\left(n_{1}\right)=\sqrt{1+4 a n_{1}},
$$

whereas SPR suggested [10]

$$
\mu_{\rho}\left(n_{1}\right)=\frac{1+3 a n_{1}}{\sqrt{1+2 a n_{1}}} .
$$

Here we calculate the chemical potential $\mu_{\rho}\left(n_{1}\right)$ of the three approaches and compare it with the precise results for $\mu_{\rho}\left(n_{1}\right)$ obtained from a numerical solution of (7). (All numerical results presented in this paper are obtained with the imaginary-time propagation scheme after a discretization by the Crank-Nicholson method using the FORTRAN programs provided in [22], the details of which are described there. The numerical simulations for the dynamical breathing oscillation and free expansion for a Fermi superfluid at unitarity reported in sections 3.3 and 3.4, respectively, were performed with the real-time propagation scheme after a discretization by the Crank-Nicholson method.) Our finding is exhibited in table 1 for different $a n_{1}$ and for $\alpha=0.985$ together with those obtained from the MMD [11] and SPR [10] schemes.

\subsection{D-2D reduction for a disc-shaped BEC}

For a disc-shaped trap the adiabatic approximation leads to the factorization $[10,11]$

$$
\psi(\mathbf{r}, t)=\varphi(\rho, t) \phi\left(z, n_{2}(\rho, t)\right),
$$

where the surface density $n_{2}$ is defined as $n_{2}(\rho, t) \equiv$ $N|\varphi(\rho, t)|^{2}=N \int \mathrm{d} z|\psi|^{2}$ and normalizations $\int \mathrm{d}^{2} \rho$ $|\varphi(\rho, t)|^{2}=\int \mathrm{d} z\left|\phi\left(z, n_{2}\right)\right|^{2}=1$. The substitution of (14)

$$
\begin{aligned}
& \text { into (2) leads to } \\
& \begin{array}{l}
\phi\left(z, n_{2}\right)\left[\mathrm{i} \frac{\partial}{\partial t}+\frac{1}{2} \nabla_{\rho}^{2}-\frac{1}{2} \rho^{2}\right] \varphi(\rho, t) \\
\quad=\varphi(\rho, t)\left[-\frac{1}{2} \frac{\partial^{2}}{\partial z^{2}}+\frac{1}{2} \lambda^{2} z^{2}+4 \pi a n_{2}|\phi|^{2}\right] \phi\left(z, n_{2}\right)
\end{array}
\end{aligned}
$$

Multiplying (15) by $\phi^{*}\left(z, n_{2}\right)$ and integrating in $z$, this equation can be rewritten as the set of equations

$$
\begin{aligned}
& {\left[\mathrm{i} \frac{\partial}{\partial t}+\frac{1}{2} \nabla_{\rho}^{2}-\frac{1}{2} \rho^{2}-\mu_{z}\left(n_{2}\right)\right] \varphi(\rho, t)=0,} \\
& {\left[-\frac{1}{2} \frac{\partial^{2}}{\partial z^{2}}+\frac{\lambda^{2} z^{2}}{2}+4 \pi a n_{2}|\phi|^{2}-\mu_{z}\left(n_{2}\right)\right] \phi\left(z, n_{2}\right)=0,} \\
& \mu_{z}\left(n_{2}\right)=\int \mathrm{d} z \phi^{*}\left[-\frac{1}{2} \frac{\partial^{2}}{\partial z^{2}}+\frac{\lambda^{2} z^{2}}{2}+4 \pi a n_{2}|\phi|^{2}\right] \phi .
\end{aligned}
$$

It is convenient to introduce scaled variables $\bar{z}=z / a_{z}, \bar{\phi}=$ $\sqrt{a}_{z} \phi$ and $\bar{\mu}_{z}=\mu_{z} a_{z}^{2}$ with $a_{z}=\sqrt{1 / \lambda}$, when (17) and (18) respectively become

$$
\left[-\frac{1}{2} \frac{\partial^{2}}{\partial \bar{z}^{2}}+\frac{\bar{z}^{2}}{2}+4 \pi a a_{z} n_{2}|\bar{\phi}|^{2}-\bar{\mu}_{z}\left(n_{2}\right)\right] \bar{\phi}\left(z, n_{2}\right)=0,
$$

$\bar{\mu}_{z}\left(n_{2}\right)=\int \mathrm{d} \bar{z} \bar{\phi}^{*}\left[-\frac{1}{2} \frac{\partial^{2}}{\partial \bar{z}^{2}}+\frac{\bar{z}^{2}}{2}+4 \pi a a_{z} n_{2}|\bar{\phi}|^{2}\right] \bar{\phi}$.

The form of the chemical potential $\bar{\mu}_{z}\left(n_{2}\right)$ of (19) is known in the small and large $N$ limits. In the small $N$ weak-coupling limit the wave-function can be approximated by the following normalized Gaussian form [21]:

$$
\bar{\phi}\left(\bar{z}, n_{2}\right)=\exp \left[-\bar{z}^{2} /\left(2 \eta^{2}\right)\right] /\left(\eta^{2} \pi\right)^{1 / 4},
$$

where $\eta$ is the width. With this wave form the chemical potential of (20) becomes

$$
\bar{\mu}_{z}\left(n_{2}\right)=\left(\frac{\eta^{2}}{4}+\frac{1}{4 \eta^{2}}\right)+2 a a_{z} n_{2} \frac{\sqrt{2 \pi}}{\eta} .
$$

In the large $N$ limit the normalization condition of the TF wave-function leads to [11]

$$
\bar{\mu}_{z}\left(n_{2}\right)=\left(3 \pi a a_{z} n_{2} / \sqrt{2}\right)^{2 / 3} .
$$

For a disc-shaped BEC we suggest the following simple interpolation formula for $\bar{\mu}_{z}\left(n_{2}\right)$ incorporating the limiting values (22) and (23):

$\bar{\mu}_{z}\left(n_{2}\right)=\frac{1}{4 \eta^{2}}-\frac{(\pi-1) \eta^{2}}{4}+\left[\left(\frac{\pi \eta^{2}}{4}\right)^{3 / 2}+\frac{3 \pi a a_{z} n_{2}}{\sqrt{2}}\right]^{2 / 3}$

valid for all $a a_{z} n_{2}$, where $\eta$ is taken as a fixed constant for all $a a_{z} n_{2}$. The flexibility in introducing a width $\eta$ slightly different from 1 in (24) will make the 2D model equation (16) an accurate approximation to the 3D GP equation (2) for a disc-shaped condensate for all $a a_{z}^{2} n_{2}$.

Instead of taking $\eta$ as a constant, SPR [10] solve (19) variationally $[10,21]$ with the Gaussian ansatz (21) and determine the width parameter $\eta$ via

$$
\eta^{4}-2 \sqrt{2 \pi} \eta a a_{z} n_{2}-1=0 \text {. }
$$


Table 2. Chemical potential $\bar{\mu}_{z}\left(n_{2}\right)$ of (20) for different $a a_{z} n_{2}$ obtained from an accurate numerical solution of (19), and from (24) (present, $\eta=0.95$ ), (26) (MMD) [11] and (25) (SPR) [10].

\begin{tabular}{cllll}
\hline$a a_{z} n_{2}$ & Numerical & MMD & SPR & Present \\
\hline 0 & 0.5 & 0.5 & 0.5 & 0.50263 \\
0.2 & 1.348783 & 1.31186 & 1.36149 & 1.34376 \\
1 & 3.599892 & 3.50165 & 3.69266 & 3.54355 \\
10 & 16.45405 & 16.2547 & 17.0012 & 16.3294 \\
100 & 76.30080 & 75.9963 & 78.8855 & 76.1358 \\
\hline
\end{tabular}

The solution of the non-linear (25) when substituted into (22) yields the desired $\bar{\mu}_{z}$ [10] through a procedure far more complicated than the analytic formulae (24).

The approximation scheme of MMD is quite involved but does not require the solution of a non-linear variational equation such as SPR. They calculate $\bar{\mu}_{z}\left(n_{2}\right)$ via [11]

$\bar{\mu}_{z}\left(n_{2}\right)=\frac{1}{8}\left[\left(\eta+\sqrt{\eta^{2}-\zeta^{6}}\right)^{1 / 3}+\left(\eta-\sqrt{\eta^{2}-\zeta^{6}}\right)^{1 / 3}-\zeta\right]^{2}$

where $\eta=4+6 \zeta-\zeta^{3}+24 \pi x$ and $\zeta=(\kappa-1)$ with $\kappa^{-1}=\sqrt{2 / \pi}+\Theta(x-0.1)(1-\sqrt{2 / \pi})\left[1-(10 x)^{-1 / 5}\right]$

where $x \equiv a a_{z} n_{2}$, and the Heaviside step function $\Theta(x-$ $0.1)=0$, for $x<0.1$, and $=1$ for $x>0.1$. It is realized that function (27) is not analytic in $x$.

Now we calculate the chemical potential $\bar{\mu}_{z}\left(n_{2}\right)$ of the three approaches and compare them with the precise result for $\bar{\mu}_{z}\left(n_{2}\right)$ from a numerical solution of (19). The results are shown in table 2 for different $a a_{z} n_{2}$ along with those from the MMD and SPR schemes. After a small experimentation the constant $\eta$ in (24) was fixed at $\eta=0.95$ for all $a a_{z} n_{2}$.

\section{3D-1D and 3D-2D reductions of Fermi superfluid DF equations}

We consider a Gallilei-invariant density-functional (DF) formulation for a trapped Fermi superfluid at BCS and unitarity limits described by $[6,7]$

$$
\left[-\mathrm{i} \hbar \frac{\partial}{\partial \hat{t}}-\frac{\hbar^{2} \nabla_{\hat{\mathbf{r}}}^{2}}{4 m}+2 V(\hat{\mathbf{r}})+2^{2 / 3} \chi \frac{2 \hbar^{2}}{m}|\Psi(\mathbf{r})|^{4 / 3}\right] \Psi(\hat{\mathbf{r}}, t)=0,
$$

with $\chi=\left(3 \pi^{2}\right)^{2 / 3} \xi / 2(\xi=1$ at the BCS limit and $\xi=0.44$ at unitarity [23]), $N$ the number of fermions, and normalization $\int|\Psi|^{2} \mathrm{~d}^{3} \hat{\mathbf{r}}=N / 2\left(|\Psi|^{2}\right.$ is the density of Fermi pairs $), m$ the mass of an atom, and $V(\hat{\mathbf{r}})=m \omega^{2}\left(\hat{\rho}^{2}+\lambda^{2} \hat{z}^{2}\right) / 2$ the harmonic trapping potential with frequencies $\omega$ and $\lambda \omega$ in radial $(\hat{\rho})$ and axial $(\hat{z})$ directions $(\hat{\mathbf{r}} \equiv \hat{\rho}, \hat{z})$ respectively. The fully paired Fermi superfluid is assumed to be composed of spin-half fermions with an equal number of spin-up and -down components. Employing dimensionless units $t=$ $\hat{t} \omega, \mathbf{r}=\hat{\mathbf{r}} / a_{\rho}, z=\hat{z} / a_{\rho}, \rho=\hat{\rho} / a_{\rho}, a=\hat{a} / a_{\rho}, \psi \sqrt{N / 2}=$ $\Psi a_{\rho}^{3 / 2}, a_{\rho}=\sqrt{\hbar / m \omega},(28)$ can be written as $\left[-\mathrm{i} \frac{\partial}{\partial t}-\frac{\nabla_{\mathbf{r}}^{2}}{4}+\rho^{2}+\lambda^{2} z^{2}+2 \chi N^{2 / 3}|\psi|^{4 / 3}\right] \psi(\mathbf{r}, t)=0$,

with $\int|\psi|^{2} \mathrm{~d}^{3} \mathbf{r}=1\left(|\psi|^{2}\right.$ is the density of Fermi atoms).

\subsection{D-1D reduction for a cigar-shaped Fermi superfluid}

For a cigar-shaped trap, we consider factorization (3). We substitute (3) into (29) and multiply the resultant equation by $\varphi^{*}\left(\rho, n_{1}\right)$ and integrate in $\rho$ to get

$$
\left[\mathrm{i} \frac{\partial}{\partial t}+\frac{1}{4} \frac{\partial^{2}}{\partial z^{2}}-\lambda^{2} z^{2}-\mu_{\rho}\left(n_{1}\right)\right] \phi(z, t)=0,
$$

where $\mu_{\rho}\left(n_{1}\right)$ is the chemical potential emerging from the following $2 \mathrm{D} \mathrm{DF}$ equation:

$$
\left[-\frac{\nabla_{\rho}^{2}}{4}+\rho^{2}+2 \chi n_{1}^{2 / 3}|\varphi|^{4 / 3}-\mu_{\rho}\left(n_{1}\right)\right] \varphi\left(\rho, n_{1}\right)=0 \text {. }
$$

In the small $N$ weak-coupling limit, the wavefunction $\varphi\left(\rho, n_{1}\right)$ can be approximated by the normalized Gaussian form [21] (8). With this wave form the chemical potential of (31) becomes

$$
\mu_{\rho}\left(n_{1}\right)=\left(\alpha^{2}+\frac{1}{4 \alpha^{2}}\right)+\frac{6 \chi}{5} \frac{n_{1}^{2 / 3}}{\alpha^{4 / 3} \pi^{2 / 3}} .
$$

The chemical potential $\mu_{\rho}\left(n_{1}\right)$ of (32) is consistent with (4.7) of AS [20]. In the large $N$ TF limit the normalization condition of the TF wave-function leads to $[10,11]$

$\mu_{\rho}\left(n_{1}\right)=\left[\left(\frac{5 n_{1}}{2 \pi}\right)^{2 / 3} 2 \chi\right]^{3 / 5} \approx 1.38336 n_{1}^{2 / 5} \chi^{3 / 5}$.

Chemical potential (33) is approximately equal to the chemical potential in the large $N$ limit of the corresponding model (4.10) of AS [20] which yields in present notation

$$
\mu_{\rho}\left(n_{1}\right)=\frac{7}{5} \frac{(6 \chi)^{3 / 5} n_{1}^{2 / 5}}{\left(5 \pi^{2}\right)^{1 / 5} 2^{2 / 5}} \approx 1.42545 n_{1}^{2 / 5} \chi^{3 / 5} .
$$

Here we use the following simple interpolation formula for $\mu_{\rho}\left(n_{1}\right)$ incorporating the limiting values (32) and (33):

$\mu_{\rho}\left(n_{1}\right)=\frac{1}{4 \alpha^{2}}-\frac{3 \alpha^{2}}{2}+\left[\left(\frac{5 \alpha^{2}}{2}\right)^{5 / 3}+2 \chi\left(\frac{5 n_{1}}{2 \pi}\right)^{2 / 3}\right]^{3 / 5}$,

where $\alpha$ is taken as a fixed constant for all $n_{1}$.

AS [20] solve equation (31) variationally and obtain for the width $\alpha$ :

$$
\alpha^{4}=\frac{1}{4}+\frac{12 \chi}{25}\left(\frac{n_{1} \alpha}{\pi}\right)^{2 / 3}
$$

Here we calculate the chemical potential $\mu_{\rho}\left(n_{1}\right)$ obtained from (35) for different $\alpha$ and from (4.7) and (4.8) of [20] and compare it with the precise result for $\mu_{\rho}\left(n_{1}\right)$ from a numerical solution of (31). Our finding is exhibited in table 3 for different $n_{1}$ together with those from the AS [20] scheme. After a small experimentation it was found that the best overall result from (35) was found for $\alpha=0.98 / \sqrt{2}$.

Now to see how well the effective 1D equations (30) and (35) reproduce the linear density $\phi^{2}(z)$ of a cigar-shaped Fermi superfluid we plot in figure 1(a) the 1D density calculated from (30) and (35) and the same calculated from the full 3D (29) for $\lambda=1 / 4$ and $N=2,10,100$. The excellent agreement between the two sets of results for $\lambda$ as large as $1 / 4$ demonstrates the usefulness of the present $1 \mathrm{D}$ model equations. 

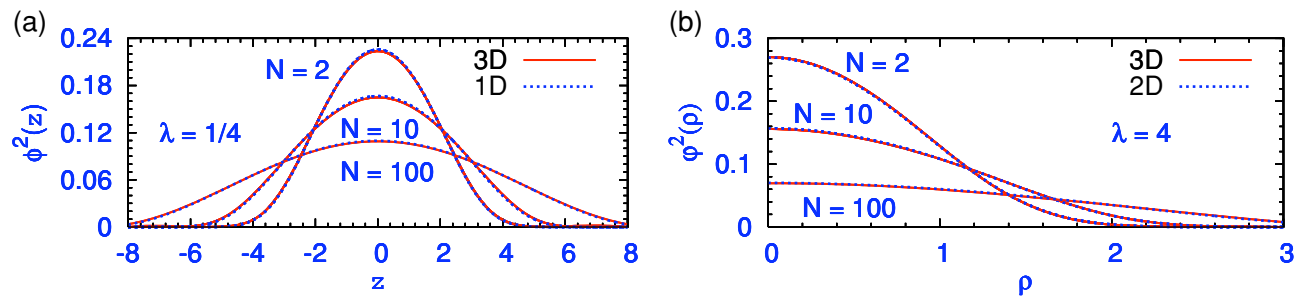

Figure 1. (a) The linear density $\phi^{2}(z)$ of a Fermi superfluid at unitarity (with $\xi=0.44$ ) versus $z$ (both in dimensionless units) calculated from the 3D DF equation (29) and the 1D model (30) and (35) for $\lambda=1 / 4, \alpha=0.98 / \sqrt{2}$ and $N=2,10$ and 100 . (b) The radial density $\varphi^{2}(\rho)$ of a Fermi superfluid at unitarity (with $\xi=0.44$ ) versus $\rho$ (both in dimensionless units) calculated from the 3D DF equation (29) and the 2D model (37) and (44) for $\lambda=4, \eta=0.92 / \sqrt{2}$ and $N=2,10$ and 100.

Table 3. Chemical potential $\mu_{\rho}\left(n_{1}\right)$ of (30) for different $n_{1}$ for a Fermi superfluid at unitarity $(\xi=0.44)$ obtained from an accurate numerical solution of (31), from (32) and (36) (AS) [6] and from (35) (present, $\alpha=1 / \sqrt{2}$ and $0.98 / \sqrt{2}$ ).

\begin{tabular}{ccccc}
\hline$n_{1}$ & Numerical & AS & $\begin{array}{l}\text { Present } \\
\alpha=1 / \sqrt{2}\end{array}$ & $\begin{array}{l}\text { Present } \\
\alpha=0.98 / \sqrt{2}\end{array}$ \\
\hline 0 & 1 & 1 & 1 & 1.00081 \\
0.1 & 1.37401 & 1.37619 & 1.36784 & 1.37667 \\
1 & 2.43893 & 2.46380 & 2.39751 & 2.41810 \\
10 & 5.56376 & 5.69170 & 5.45891 & 5.49147 \\
100 & 13.70413 & 14.09704 & 13.5463 & 13.5867 \\
1000 & 34.29934 & 35.33109 & 34.1057 & 34.1506 \\
\hline
\end{tabular}

\subsection{D-2D reduction for a disc-shaped Fermi superfluid}

In the case of a disc-shaped trap we consider the factorization (14) and substitute it into (29), multiply the resultant equation by $\phi^{*}\left(z, n_{2}\right)$ and integrate in $z$ to get

$$
\left[i \frac{\partial}{\partial t}+\frac{1}{4} \nabla_{\rho}^{2}-\rho^{2}-\mu_{z}\left(n_{2}\right)\right] \varphi(\rho, t)=0
$$

where $\mu_{z}\left(n_{2}\right)$ is the chemical potential emerging from the following $1 \mathrm{D}$ DF equation:

$\left[-\frac{1}{4} \frac{\partial^{2}}{\partial z^{2}}+\lambda^{2} z^{2}+2 \chi n_{2}^{2 / 3}|\phi|^{4 / 3}-\mu_{z}\left(n_{2}\right)\right] \phi\left(z, n_{2}\right)=0$.

It is convenient to introduce scaled variables $\bar{z}=z / a_{z}, \bar{\phi}=$ $\sqrt{a}_{z} \phi$ and $\bar{\mu}_{z}=\mu_{z} a_{z}^{2}$ with $a_{z}=\sqrt{1 / \lambda}$, when (38) becomes

$$
\left[-\frac{1}{4} \frac{\partial^{2}}{\partial \bar{z}^{2}}+\bar{z}^{2}+2 \chi a_{z}^{4 / 3} n_{2}^{2 / 3}|\bar{\phi}|^{4 / 3}-\bar{\mu}_{z}\left(n_{2}\right)\right] \bar{\phi}\left(z, n_{2}\right)=0 \text {, }
$$

$\bar{\mu}_{z}\left(n_{2}\right)=\int \mathrm{d} \bar{z} \bar{\phi}^{*}\left[-\frac{1}{4} \frac{\partial^{2}}{\partial \bar{z}^{2}}+\bar{z}^{2}+2 \chi a_{z}^{4 / 3} n_{2}^{2 / 3}|\bar{\phi}|^{4 / 3}\right] \bar{\phi}$.

The form of the chemical potential $\bar{\mu}_{z}\left(n_{2}\right)$ of (39) is known in the small and large $N$ limits. In the small $N$ weak-coupling limit the wave-function $\bar{\phi}\left(\bar{z}, n_{2}\right)$ can be approximated by the normalized Gaussian form [21] (21). With this wave form the chemical potential of (40) becomes

$$
\bar{\mu}_{z}\left(n_{2}\right)=\left(\frac{\eta^{2}}{2}+\frac{1}{8 \eta^{2}}\right)+\frac{2 \chi\left(n_{2} a_{z}^{2}\right)^{2 / 3}}{\eta^{2 / 3} \pi^{1 / 3}} \sqrt{\frac{3}{5}} .
$$

The chemical potential $\bar{\mu}_{z}\left(n_{2}\right)$ of (41) is consistent with (5.7) of AS [20]. In the large $N$ TF limit the normalization condition of the TF wave-function leads to [11]

$$
\bar{\mu}_{z}\left(n_{2}\right)=\left[\frac{8 \chi n_{2}^{2 / 3} a_{z}^{4 / 3}}{(3 \pi)^{2 / 3}}\right]^{3 / 4} \approx 1.54947 \chi^{3 / 4} a_{z} \sqrt{n_{2}} .
$$

Chemical potential (42) is approximately equal to the chemical potential in the large $N$ limit of the corresponding model (5.10) of AS [20] which yields in the present notation

$$
\bar{\mu}_{z}\left(n_{2}\right)=\frac{12 a_{z} 3^{3 / 8} \chi^{3 / 4} \sqrt{n_{2}}}{5 \sqrt{2} \pi^{1 / 4} 5^{1 / 8}} \approx 1.57383 \chi^{3 / 4} a_{z} \sqrt{n}_{2} .
$$

Here we use the following interpolation formula for $\bar{\mu}_{z}\left(n_{2}\right)$ incorporating the limiting values (41) and (42):

$$
\begin{aligned}
\bar{\mu}_{z}\left(n_{2}\right) & =\left(\frac{\eta^{2}}{2}+\frac{1}{8 \eta^{2}}\right)-\frac{15^{3 / 2} \eta^{2}}{9 \pi} \\
+ & {\left[\left(\frac{15^{3 / 2} \eta^{2}}{9 \pi}\right)^{4 / 3}+8 \chi \frac{\left(n_{2} a_{z}^{2}\right)^{2 / 3}}{(3 \pi)^{2 / 3}}\right]^{3 / 4}, }
\end{aligned}
$$

where $\eta$ is taken as a fixed constant for all $a_{z}^{2} n_{2}$.

AS [20] solve equation (39) variationally and obtain for the width $\eta$ :

$$
\eta^{4}=\frac{1}{4}+\frac{4 \chi}{5} \frac{\left(a_{z}^{2} n_{2} \eta^{2}\right)^{2 / 3}}{\pi^{1 / 3}} \sqrt{\frac{3}{5}} .
$$

As in the 3D-1D reduction, now we calculate the chemical potential $\bar{\mu}_{z}\left(n_{2}\right)$ of the three approaches and compare them with the precise result for $\bar{\mu}_{z}\left(n_{2}\right)$ obtained from a numerical solution of (39). The results are shown in table 4 for different $a_{z}^{2} n_{2}$ along with those from the AS scheme [20].

Now to see how well the effective 2D equations (37) and (44) reproduce the radial density $\varphi^{2}(\rho)$ of a disc-shaped Fermi superfluid we plot in figure 1 (b) the 2D density calculated from (37) and (44) and the same calculated from the full 3D (29) for $\lambda=4$ and $N=2,10,100$. The excellent agreement between the two sets of results for $\lambda$ as small as 4 demonstrates the usefulness of the present 2D model equations.

\subsection{Dynamics: breathing oscillation}

Now we subject the reduced 1D and 2D DF equations to a more stringent test, e.g. how well these equations can reproduce nonstationary (non-equilibrium) dynamics of a cigar- and discshaped Fermi superfluid. First we consider a cigar-shaped 

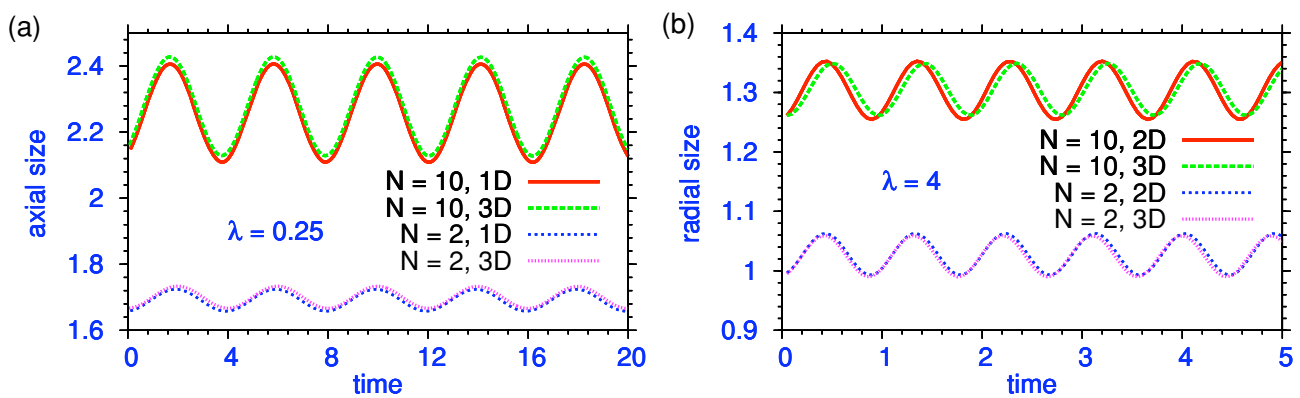

Figure 2. (a) The rms axial size versus time (both in dimensionless oscillator units) during oscillation of a cigar-shaped Fermi superfluid at unitarity $(\xi=0.44)$ for $\lambda=0.25$, started by reducing the axial trap suddenly by a factor of 0.9 , as calculated by the full 3D equation (29) and the reduced 1D equations (30) and (35) for $\alpha=0.98 / \sqrt{2}$. (b) The rms radial size versus time (both in dimensionless oscillator units) during oscillation of a disc-shaped Fermi superfluid at unitarity for $\lambda=4$, started by reducing the radial trap suddenly by a factor of 0.9 , as calculated by the full 3D equation (29) and the reduced 2D equations (37) and (44) for $\eta=0.92 / \sqrt{2}$.
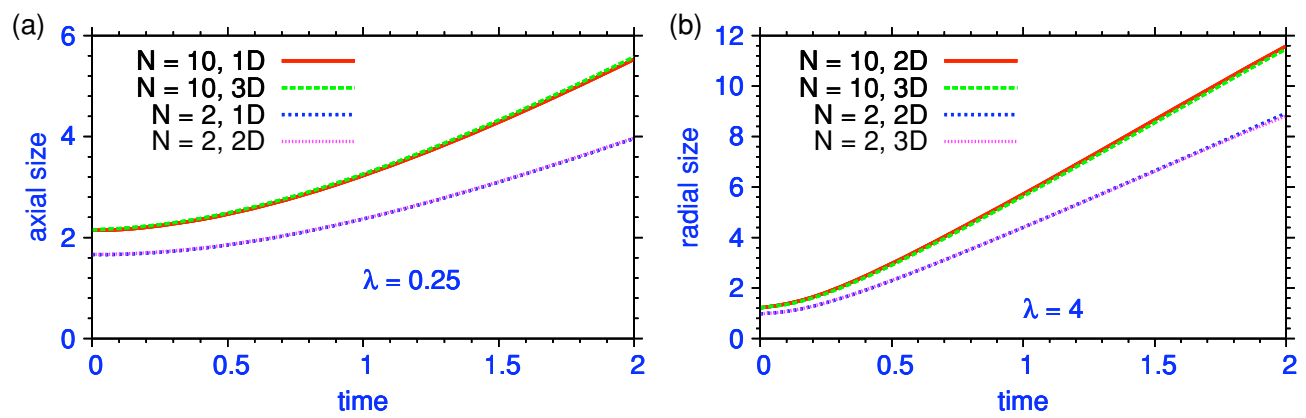

Figure 3. (a) The rms axial size versus time (both in dimensionless oscillator units) during axial expansion of a cigar-shaped Fermi superfluid at unitarity $(\xi=0.44)$ for $\lambda=0.25$, started by removing the axial trap suddenly, as calculated by the full 3D equation (29) and the reduced 1D equations (30) and (35) for $\alpha=0.98 / \sqrt{2}$. (b) The rms radial size versus time (both in dimensionless oscillator units) during radial expansion of a disc-shaped Fermi superfluid at unitarity for $\lambda=4$, started by removing the radial trap suddenly, as calculated by the full 3D equation (29) and the reduced 2D equations (37) and (44) for $\eta=0.92 / \sqrt{2}$.

Table 4. Chemical potential $\bar{\mu}_{z}\left(n_{2}\right)$ of (40) for different $a_{z}^{2} n_{2}$ for a Fermi superfluid at unitarity $(\xi=0.44)$ obtained from an accurate numerical solution of (39), from (41) and (45) (AS) [6] and from (44) (present, $\eta=1 / \sqrt{2}$ and $\eta=0.92 / \sqrt{2}$ ).

\begin{tabular}{cllll}
\hline$a_{z}^{2} n_{2}$ & Numerical & AS & $\begin{array}{l}\text { Present } \\
\eta=1 / \sqrt{2}\end{array}$ & $\begin{array}{l}\text { Present } \\
\eta=0.92 / \sqrt{2}\end{array}$ \\
\hline 0 & 0.5 & 0.5 & 0.5 & 0.50696 \\
0.1 & 1.071781 & 1.07375 & 1.05892 & 1.08883 \\
1 & 2.79861 & 2.82290 & 2.72155 & 2.78109 \\
10 & 8.59782 & 8.72108 & 8.41456 & 8.50454 \\
100 & 27.09534 & 27.51645 & 26.8149 & 26.9282 \\
500 & 60.56659 & 61.51645 & 60.2317 & 60.3571 \\
\hline
\end{tabular}

Fermi superfluid with $\lambda=0.25$, which is set into breathing oscillation by reducing only the axial potential suddenly by a factor of 0.9. The radial trap is left unchanged. The resultant oscillation is studied using the full 3D DF equation (29) as well as the reduced 1D DF equation (30) using the chemical potential (35). The root mean square (rms) axial size as calculated from the $3 \mathrm{D}$ and $1 \mathrm{D}$ equations is plotted in figure 2(a). Next we consider a disc-shaped Fermi superfluid with $\lambda=4$, which is set into breathing oscillation by reducing only the radial potential suddenly by a factor of 0.9. The resultant oscillation is studied using the full 3D DF equation (29) as well as the reduced 2D DF equation (37) using the chemical potential (44). The rms radial size as calculated from the $3 \mathrm{D}$ and $2 \mathrm{D}$ equations is plotted in figure 2(b).

\subsection{Dynamics: free expansion}

Now we consider the problem of free expansion of a cigar- and disc-shaped Fermi superfluid, respectively, when the axial and radial traps are suddenly removed after the formation of the superfluid. First we consider a cigar-shaped Fermi superfluid with $\lambda=0.25$ which is allowed to expand freely in the axial direction by setting the axial trap suddenly to zero in the $3 \mathrm{D}$ equation. The radial trap is left unchanged. The resultant expansion is studied using the full 3D DF equation (29) as well as the reduced 1D DF equation (30) using the chemical potential (35). The rms axial size as calculated from the 3D and 1D equations are plotted in figure 3(a). Next we consider a disc-shaped Fermi superfluid with $\lambda=4$, which is allowed to expand freely in the radial direction by setting the radial trap suddenly to zero in the $3 \mathrm{D}$ equation. The axial trap is left unchanged. The resultant expansion is studied using the full 3D DF equation (29) as well as the reduced 2D DF equation (37) using the chemical potential (44). The rms radial size as calculated from the $3 \mathrm{D}$ and $2 \mathrm{D}$ equations is plotted in figure 3(b). The agreement between the dynamics as obtained 
from the full 3D DF equation and that from the reduced DF equations shown in figures 2 and 3 is quite satisfactory.

\section{Conclusion}

In conclusion, we have suggested time-dependent mean-field reduced $\mathrm{DF}$ equations in $1 \mathrm{D}$ and $2 \mathrm{D}$, respectively, for a cigarand disc-shaped BEC and Fermi superfluid in the BCS and unitarity limits with simple analytic non-linear terms. The numerical solution of these reduced equations reveals that they produce results for the density of Fermi superfluids and BEC in cigar- and disc-shaped traps in excellent agreement with the solution of the full 3D DF equation. We also studied nonstationary breathing oscillation of the cigar- and disc-shaped Fermi superfluid initiated by a sudden change of axial and radial traps, respectively. Finally, we applied the reduced equations to the study of free expansion of a cigar- and discshaped Fermi superfluid initiated by a sudden removal of the axial and radial trap, respectively. The reduced equations produced equally good results in both these studies when compared with the solution of the full $3 \mathrm{D}$ equations.

\section{Acknowledgments}

FAPESP, CAPES and CNPq (Brazil) provided partial support.

\section{References}

[1] Dalfovo F, Giorgini S, Pitaevskii L and Stringari S 1999 Rev. Mod. Phys. 71463

[2] Eagles D M 1969 Phys. Rev. 186456

Leggett A J 1980 J. Phys. (Paris) Colloq. 41 C7-19

Adhikari S K et al 2000 Phys. Rev. B 628671
[3] Giorgini S, Pitaevskii L and Stringari S 2008 Rev. Mod. Phys. 801215

[4] Greiner M, Regal C A and Jin D S 2003 Nature 426537 Kinast J, Hemmer S L, Gehm M E, Turlapov A and Thomas J E 2004 Phys. Rev. Lett. 92150402

[5] Zwierlein M W et al 2004 Phys. Rev. Lett. 92120403 Zwierlein M W, Schunck C H, Stan C A, Raupach S M F and Ketterle W 2005 Phys. Rev. Lett. 94180401

[6] Adhikari S K and Salasnich L 2008 Phys. Rev. A 78043616

[7] Adhikari S K 2009 Phys. Rev. A 79023611 Adhikari S K 2008 Phys. Rev. A 77045602 Adhikari S K 2009 Laser Phys. Lett. 6901

[8] Blume D, von Stecher J and Greene C H 2007 Phys. Rev. Lett. 99233201

Chang S Y and Bertsch G F 2007 Phys. Rev. A 76021603

[9] Görlitz A et al 2001 Phys. Rev. Lett. 87130402

[10] Salasnich L, Parola A and Reatto L 2002 Phys. Rev. A 65043614

[11] Muñoz Mateo A and Delgado V 2008 Phys. Rev. A 77013617

[12] Salasnich L, Parola A and Reatto L 2004 Phys. Rev. A 69045601

[13] Muñoz Mateo A and Delgado V 2007 Phys. Rev. A 75063610

[14] Jackson A D, Kavoulakis G M and Pethick C J 1998 Phys. Rev. A 582417

[15] Chiofalo M L and Tosi M P 2000 Phys. Lett. A 268406

[16] Massignan P and Modugno M 2003 Phys. Rev. A 67023614

[17] Kamchatnov A M and Shchesnovich V S 2004 Phys. Rev. A 70023604

[18] Zhang W and You L 2005 Phys. Rev. A 71025603

[19] Abdullaev F K and Galimzyanov R 2003 J. Phys. B: At. Mol. Opt. Phys. 361099

[20] Adhikari S K and Salasnich L 2009 New J. Phys. 11023011

[21] Pérez-García V M, Michinel H, Cirac J I, Lewenstein M and Zoller P 1997 Phys. Rev. A 561424

[22] Adhikari S K and Muruganandam P 2009 Comput. Phys. Commun. 1801888

[23] Astrakharchik G E et al 2004 Phys. Rev. Lett. 93200404 Carlson J et al 2003 Phys. Rev. Lett. 91050401 